\title{
Zertifizierung im medizinischen Kontext
}

Empfehlungen der Schweizerischen Akademie der Medizinischen Wissenschaften

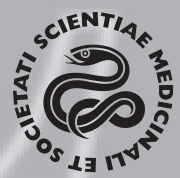

SAMW

Schweizerische Akademie

der Medizinischen

Wissenschaften

\section{ASSM}

Académie Suisse

des Sciences Médicales

\section{ASSM}

Accademia Svizzera delle

Scienze Mediche 



\section{Zertifizierung im medizinischen Kontext}

\section{Empfehlungen der Schweizerischen Akademie der Medizinischen Wissenschaften ${ }^{1}$}

I. Hintergrund 2

II. Aufgaben und Grenzen der Zertifizierung im
medizinischen Kontext

III. Empfehlungen 5

1. Das Zertifikat bestätigt einen Mehrwert 5

2. Die Zertifizierungsstelle ist für ihre Aufgabe qualifiziert 7

3. Die normativen Grundlagen der Zertifizierung sind offengelegt $\quad 8$

4. Es gibt ein Audit-Verfahren 8

5. Das Zertifikat ist zeitlich begrenzt 9

$\begin{array}{ll}\text { Anhang } & 10\end{array}$

1. Checkliste: Formale Anforderungen an ein Zertifikat 10

2. Gesetzliche Grundlagen 12

3. Glossar 13

Der Senat der Schweizerischen Akademie der Medizinischen Wissenschaften hat diese Empfehlungen am 24. Mai 2011 genehmigt. Der Arbeitsgruppe, welche diese Empfehlungen ausgearbeitet hat, gehörten folgende Personen an: Prof. Dieter Conen, Präsident Stiftung für Patientensicherheit, Buchs AG (Vorsitz); Dr. Hermann Amstad, Generalsekretär SAMW, Basel; Dr. Daniel Herren, Mitglied Zentralvorstand FMH, Zürich; Dr. Kurt Hess, Mitglied Stiftungsrat EQUAM, Zürich; Dr. Olivier Kappeler, Hausarzt, Weinfelden; Prof. Annemarie Kesselring, ehem. Extraordinaria des Instituts für Pflegewissenschaft Basel, Bern; Margrit Kessler, Präsidentin SPO Patientenschutz, Zürich; Dr. René Kühne, Helsana Versicherungen, Dübendorf; Dr. Monika Maritz Mosimann, Hausärztin, Gunten; Prof. Urs Metzger, ehem. Chefarzt Chirurgie Triemli, Zürich; Dr. Jürg Nadig, Präsident der Schweiz. Gesellschaft für Medizinische Onkologie, Bülach; lic. rer. pol. Martin Rothenbühler, Geschäftsführer SanaCERT, Bern; Prof. Christian Ruef, Leiter Spitalhygiene des UniversitätsSpitals Zürich, Zürich; Prof. Anthony Staines, Stiftungsrat SanaCERT, Clarmont; Dr. Rolf Straub, Mitarbeiter SAS, Bern Wabern; Dr. Georg von Below, Vorstandsmitglied der Swiss Society for Quality Management in Health Care (SQMH), Bern; Dr Jean-Blaise Wasserfallen, Mitglied der Spitaldirektion CHUV, Lausanne 


\section{Zertifizierung im medizinischen Kontext}

\section{Empfehlungen der Schweizerischen Akademie der Medizinischen Wissenschaften}

\section{Hintergrund}

Das Gesundheitssystem ist in Diagnostik und Therapie einem steten und raschen technologischen Wandel unterworfen. Die Vermehrung des medizinischen Wissens, die immer komplexeren und in rascherer Abfolge angebotenen Abklärungs- und Behandlungsoptionen erschweren zunehmend die Übersicht über Umfang und Nutzen der vorhandenen Angebote.

Die Forderungen nach entsprechenden Qualitätsnachweisen und vermehrter Transparenz (vgl. KVG und KVV; siehe Anhang B) haben zu verschiedenen Aktivitäten und Angeboten im Gesundheitswesen geführt; dazu gehört auch die Abgabe von Zertifikaten ${ }^{2}$. Parallel dazu führt allerdings die in den letzten Jahren wachsende Zahl von Zertifizierungsangeboten und Qualitätslabels zu Unsicherheit und Verwirrung.

Eine Analyse des Ist-Zustandes deckt denn auch zahlreiche Schwachstellen des heutigen Systems auf:

- Die Bedeutung der Zertifikate ist häufig nicht klar.

- Die Zielgruppen für die Botschaft der Zertifikate sind nicht immer klar identifiziert.

- Die Unparteilichkeit und Unabhängigkeit der Prüfenden und/oder der Entscheidenden sind fraglich.

- Die normativen Grundlagen sind nicht allgemein zugänglich.

- Die normativen Grundlagen beruhen oft nicht auf einem breit abgestützten Konsens mit Leistungserbringern und Stakeholdern bzw. nicht auf einer wissenschaftlich validierten Basis, welche die Ergebnisse der Evidenz basierten Medizin berücksichtigt.

- Die Gültigkeitsdauer der Zertifikate ist nicht immer beschränkt bzw. öffentlich sichtbar.

- Die Listen der zertifizierten Einrichtungen und Produkte bzw. Leistungen werden nicht veröffentlicht. 
Vor diesem Hintergrund hat die Verbindung der Schweizer Ärztinnen und Ärzte FMH gegenüber der Schweizerischen Akademie der Medizinischen Wissenschaften (SAMW) angeregt, diese Situation zu verbessern und mit der Ausarbeitung von breit abgestützten Empfehlungen einen Beitrag zu mehr Transparenz zu schaffen. Der Vorstand der SAMW hat diese Anregung aufgegriffen und einer Arbeitsgruppe unter Leitung von Prof. Dieter Conen einen entsprechenden Auftrag erteilt. Die folgenden Empfehlungen richten sich in erster Linie an die Leistungserbringer ${ }^{3}$ im Gesundheitswesen, an die verschiedenen interprofessionellen Teams, an die Versicherer und an die politischen Behörden.

Zertifikate, welche diesen Empfehlungen Folge leisten, können in der Regel als vertrauenswürdig und sinnvoll gelten. In diesem Sinne sind die Empfehlungen auch für die Patienten relevant: Sie sind eingeladen, bei jedem Zertifikat nach dessen Übereinstimmung mit den vorliegenden Empfehlungen zu fragen.

\section{Aufgaben und Grenzen der Zertifizierung im medizinischen Kontext}

Zertifikate im Gesundheitswesen sollen zur Verminderung einer bestehenden Informationsasymmetrie zwischen Anbietern und Nachfragern bezüglich der Qualität von Leistungen beitragen. Zusammen mit messbaren, validen und zuverlässigen Indikatoren, die allenfalls in Qualitätsberichten publiziert werden, können sie auch dazu dienen, gegenüber Dritten (z. B. Kantonen und Bund sowie Versicherungen) das Vorhandensein und Funktionieren eines Qualitätsmanagementsystems und die Erfüllung gesetzlicher Anforderungen nachzuweisen. Ausserdem zeigen sie dem Zertifizierten das Abweichen von einem Standard auf und signalisieren damit Verbesserungspotential.

Die Qualität wird entsprechend dem 2001 veröffentlichten Report «Crossing the Quality Chasm: A New Health System for the Twentyfirst Century» des US-amerikanischen Institute of Medicine folgendermassen definiert:

«Qualität ist ein Mass dafür, wie sehr Leistungen des Gesundheitswesens für Individuen oder ganze Bevölkerungsgruppen

- die Wahrscheinlichkeit erwünschter Gesundheitsergebnisse erhöhen und

- dem aktuellen Fachwissen entsprechen.»

3 Im Hinblick auf die leichtere Lesbarkeit dieses Textes wird in der Regel nur die männliche Form verwendet; selbstverständlich sind immer beide Geschlechter gemeint. 
Im Gegensatz zur blossen Behauptung, dass «die Qualität gut ist», sollten Zertifikate im Idealfall der Nachweis dafür sein, dass ein QM-System installiert ist bzw. dass Standards für Strukturen, Prozesse und Ergebnisse der Leistungserbringung definiert und diese qualitätswirksam (also die Qualität der Leistungen beeinflussend) sind.

Ziel des Qualitätsprozesses muss es sein, ein gutes Ergebnis zu erzielen, und auch die Informationsasymmetrie zu verkleinern, indem für Patienten, ihre Angehörigen und für die verschiedenen Stakeholder die angebotene Qualität transparent und nachvollziehbar dokumentiert und verständlich dargelegt wird.

\section{Zertifizierung braucht das Engagement der Leitungsebene}

$\mathrm{Ob}$ und welche Zertifizierung eine Einrichtung erwerben soll, muss Gegenstand qualitätsstrategischer Überlegungen der obersten Entscheidungsinstanz einer Einrichtung im Gesundheitswesen bilden. Ein unkoordiniertes Ansammeln verschiedenster Zertifikate ohne entsprechende Dachstrategie ist kostspielig und wenig sinnvoll. Nicht zuletzt auch aus diesem Grund ist es nötig, dass die zu zertifizierende Organisation über ein Qualitätsmanagementsystem verfügt, das die verschiedenen Verantwortlichkeiten system- und stufengerecht und mit den unterschiedlichen Aufgaben und Pflichten verbunden festlegt.

\section{Zertifizierung ersetzt nicht einen eigentlichen Qualitätsnachweis}

Zertifikate sind keine Garantie für Qualität. Es gibt dementsprechend auch keine generelle Evidenz dafür, dass Zertifikate zwingend bessere End-Qualität (Outcome) sicherstellen. Zertifikate können jedoch eine Aussage darüber machen, wie gut die Voraussetzungen in einer Organisation sind, Qualität zu produzieren. Aus diesem Grund sehen moderne Zertifizierungssysteme als integralen Bestandteil Kontrollen dazu vor, ob Qualitätsmessungen (z.B. über Indikatorensysteme) durchgeführt werden. Damit sollen einerseits die Qualitätsnachweise überprüft und andererseits die Wirksamkeit von repetitiven Zertifizierungsverfahren und der Zertifikate dokumentiert werden. 
Eine Zertifizierung ist dann angezeigt, wenn ein zusätzlicher Nutzen nach Innen oder nach Aussen zu erwarten ist:

Innerhalb der Organisation sollen Anhaltspunkte gefunden werden, die zu einer Unterstützung der internen Qualitätsverbesserungsmassnahmen beitragen Durch den Vergleich mit einem Standard kann die Organisation erkennen, wo das Verbesserungspotential liegt. Ausserdem werden idealerweise alle im QM-System betroffenen Ebenen einbezogen, so dass eine Beurteilung des Gesamtsystems möglich ist.

Nach Aussen bewirkt die Zertifizierung eine gute Sichtbarkeit des Leistungsangebotes und idealerweise der Ergebnisse der erbrachten Leistungen für nach Orientierung suchende Patienten, für die Krankenversicherer, aber auch für die politischen Behörden und nicht zuletzt für potenzielle Mitarbeiter. Eine nach transparenten Kriterien durchgeführte Zertifizierung schafft wegen der dokumentierten Validität eine nicht zu unterschätzende Reputation der zertifizierten Stelle bei Partnern und in der breiten Öffentlichkeit.

Darüber hinaus müssen Zertifikat und Zertifizierung die folgenden formalen Kriterien erfüllen.

\section{Das Zertifikat bestätigt einen Mehrwert.}

Es muss nachvollziehbar sein, was der Mehrwert eines zertifizierten Managementsystems, eines Produktes oder einer Leistung ist gegenüber Managementsystemen, Produkten oder Leistungen, die nicht zertifiziert sind und lediglich die gesetzlich verlangten Grundvoraussetzungen formal erfüllen. Dieser Mehrwert sollte darlegbar sein und mit validen und zuverlässigen Indikatoren quantifiziert werden können, die idealerweise national und international Gültigkeit besitzen. Wenn Indikatoren für die Beurteilung der Ergebnisqualität vorliegen, ist dies ein wesentliches Merkmal einer nutzenbringenden Zertifizierung. Hier kann unterschieden werden zwischen organisationsübergreifenden Indikatoren (z.B. im stationären Bereich: Rate der nosokomialen Infekte? Häufigkeit einer nosokomialen Pneumonie, von Stürzen, von Dekubitus?) oder abteilungsbezogenen Indikatoren (Besteht eine systematische Erfassung postoperativer Wundinfekte? Wie ist die Rate der fremdkörperbedingten Infekte? Existiert ein Fehlermelde- und -bearbeitungssystem?). Auch für den am- 
bulanten Bereich sollen typische Indikatoren herangezogen werden (z.B. für die Qualität der Behandlung des Diabetes mellitus; für die Einstellung der arteriellen Hypertonie; die Messung der Patientenzufriedenheit).

Für die Organisation gilt es zu entscheiden, ob der Aufwand an Kosten, den der Zertifizierungsprozess bedeutet, einen entsprechenden Mehrwert generiert, denn neben den intern während der Arbeitszeit von den Mitarbeitern zu erbringenden Leistungen kommen eventuell noch Beraterkosten und die Kosten für die zertifizierende Organisation dazu. Gleichzeitig muss bedacht werden, dass in der Regel eine Rezertifizierung nach drei Jahren nötig ist, um zu zeigen, dass die geforderten und eingeführten Prozessoptimierungen weiterhin zur Anwendung kommen und allenfalls zur Verbesserung oder Stabilisierung der Qualitätskennzahlen resp. zu den -zielen geführt haben.

\section{Kriterien:}

- Das Zertifikat gibt an, was zertifiziert wird (z.B. Teilnahme am Qualitätsprozess; medizinische Behandlung).

- Das Zertifikat gibt an, wer mit dem Zertifikat ausgezeichnet wird ( ein einzelner ärztlicher Leistungserbringer; ein einzelner anderer Leistungserbringer im Gesundheitswesen; interprofessionelle Teams; Ärztenetzwerke; eine Abteilung innerhalb einer Institution; ein ganzes Spital bzw. eine einzelne Klinik).

- Das Zertifikat gibt Indikatoren vor, welche die Beurteilung wesentlicher diagnostischer Prozesse ermöglichen.

- Das Zertifikat gibt Indikatoren vor, welche die Beurteilung wesentlicher therapeutischer Prozesse ermöglichen.

- Das Zertifikat gibt Indikatoren vor, welche die Beurteilung der Behandlungsergebnisse ermöglichen.

- Das Zertifikat gibt Indikatoren vor, welche einen relevanten Vergleich auf nationaler und wenn möglich auf internationaler Ebene ermöglichen.

- Das Zertifikat bestätigt das Vorhandensein einzuhaltender diagnostischer Standards.

- Das Zertifikat bestätigt das Vorhandensein einzuhaltender therapeutischer Standards. 
Die Akkreditierung ist das international anerkannte Mittel, um Vertrauen und Zuverlässigkeit in die Zertifizierung zu bringen.

Die Akkreditierung bestätigt, dass eine zertifizierende Stelle für ihre Tätigkeit die nötige Kompetenz besitzt, dass das Zertifizierungsverfahren fundiert, zielführend und sinnvoll ist, dass Transparenz über die zertifizierten Organisationen vorliegt. und dass die zertifizierende Stelle unabhängig ist. Das heisst, die zertifizierende Stelle hat die Fach-, Managementund Methodenkompetenz, um eine Organisation im Gesundheitswesen zu zertifizieren. Akkreditierte Stellen betreiben selbst ein Qualitätsmanagementsystem, so dass sie auch die Qualität ihrer Hauptaufgaben sichern.

Kriterien:

- Die Stelle, welche ein Zertifikat erteilt, ist bei der Schweizerischen Akkreditierungsstelle (SAS) oder einer gleichwertigen Institution akkreditiert.

- Die Zertifizierungsstelle veröffentlicht eine Liste der zertifizierten Organisationen.

3. Die normativen Grundlagen der Zertifizierung sind offengelegt.

Die normativen Anforderungen, die zu erfüllen sind, um ein Zertifikat zugesprochen zu erhalten, müssen klar definiert und für alle interessierten Parteien zugänglich sein. Normativ festgelegte Anforderungen als Basis für die Zertifizierung haben nur dann einen Sinn, wenn sie bekannt sind und von relevanten interessierten Stellen wie z.B. Kunden, Behörden und Versicherungen entsprechend eingeschätzt und gewürdigt werden. Die Voraussetzung dafür: Normative Anforderungen an Managementsysteme sind innerhalb relevanter Fachbranche(n), betroffener Verbände oder durch Behörden des Bundes bzw. der Kantone als zertifizierungswürdige Grundlage anerkannt ${ }^{4}$.

Diese breite Anerkennung kann durch ein Vernehmlassungsverfahren unter Einschluss aller interessierten Kreise erreicht werden. Der gesamte Konsens-Findungsprozess muss dokumentiert werden.

Kriterien:

- Die normativen Grundlagen für das Zertifikat beziehen die vorhandene wissenschaftliche Evidenz mit ein. 
- Die normativen Grundlagen beruhen auf einem breiten fachlichen Konsens.

- Die normativen Grundlagen sind kostenlos zugänglich.

- Die Gültigkeitsdauer der normativen Grundlagen ist festgelegt.

\section{Es gibt ein Audit-Verfahren.}

Eine sinnvolle Zertifizierung besteht in der Regel aus einer initialen Selbstdeklaration, welche die wesentlichen Struktur-, Prozess- und Ergebniselemente gemäss einer standardisierten Vorgabe beurteilt.

Daran schliesst sich eine Vor-Ort-Besichtigung an, bei der neben Fachleuten der Zertifizierungsstelle auch Peers mitwirken sollten. Diese Auditoren sind entsprechend geschult; sie führen Interviews, beobachten Abläufe, überprüfen das Vorhandensein von Standards und kontrollieren Indikatoren. Ihre Ausbildung ist dokumentiert und die Dokumente liegen der zu zertifizierenden Stelle vor.

Zeitnah erfolgt eine schriftliche Beurteilung mit einer detaillierten Beschreibung des Audit-Verfahrens, einer nachvollziehbaren Aufzählung der festgestellten Defizite und möglichen Empfehlungen. Beides wird der zu zertifizierenden Stelle zur Stellungnahme vorgelegt.

Ein von den Auditoren unabhängiges Gremium wird dann die Zertifizierung als bestanden, als mit Auflagen bestanden oder als nicht bestanden beurteilen.

Kriterien:

- Das Audit-Verfahren ist klar beschrieben.

- Die Rolle der Auditoren (Fachleute der Zertifizierungsstelle und Peers) ist klar definiert.

- Die Rolle der zu zertifizierenden Stelle ist klar definiert.

- Die Auditoren sind geschult.

- Die Auditoren sind unabhängig von der zu zertifizierenden Stelle. 
Ohne eine zeitliche Begrenzung der Geltungsdauer eines Zertifikats ist weder zu beurteilen, ob sich die Leistung gehalten hat, noch ob sie sich den modernen Entwicklungen angepasst hat.

Im weiteren wird auch eine kulturelle Veränderung nur im Zeitverlauf sichtbar, so dass ein Zertifikat immer nur eine Bestätigung auf Zeit sein kann.

Kriterien:

- Die Gültigkeitsdauer des Zertifikates ist angegeben.

- Die Gültigkeitsdauer des Zertifikates beträgt max. 5 Jahre. 


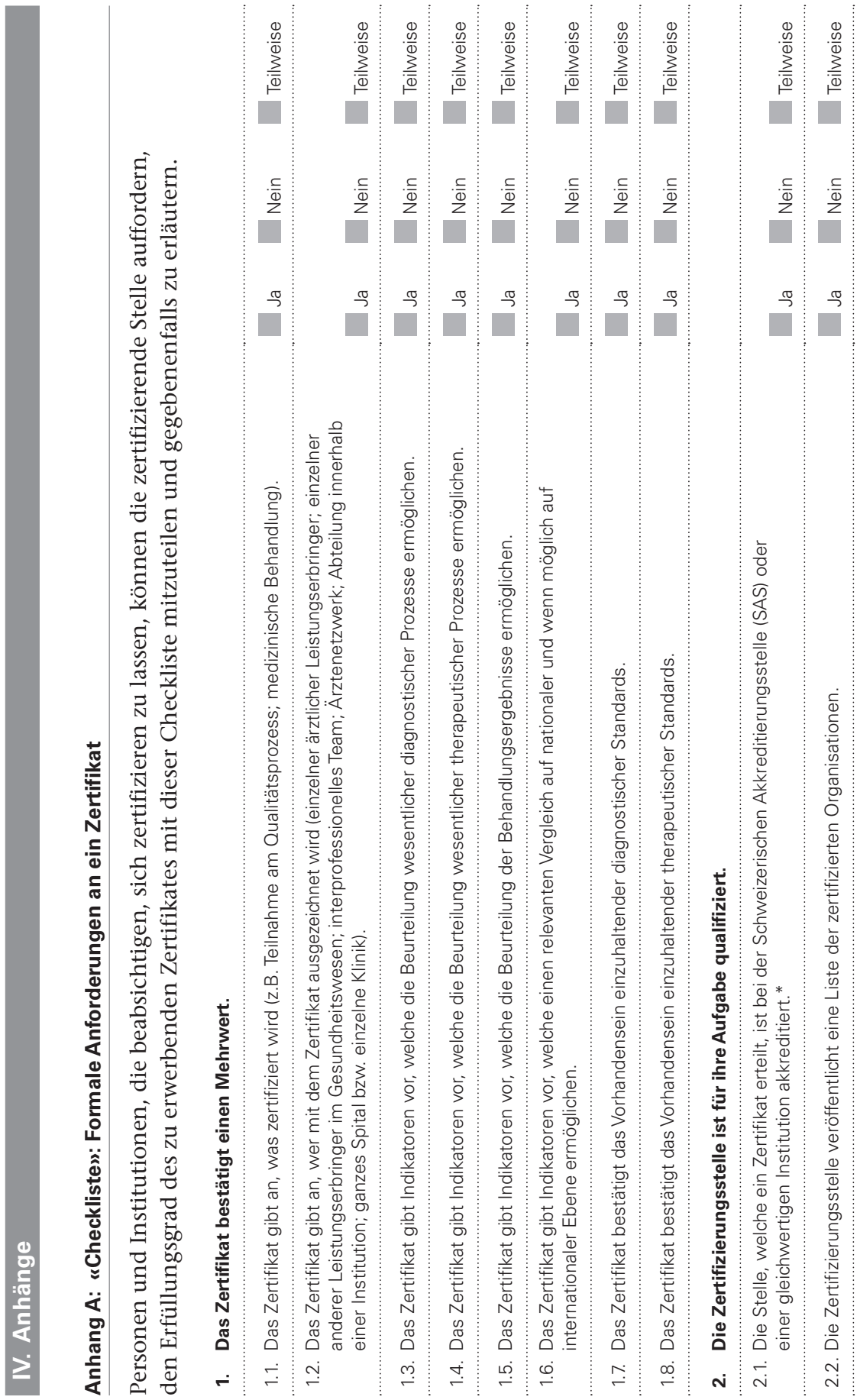




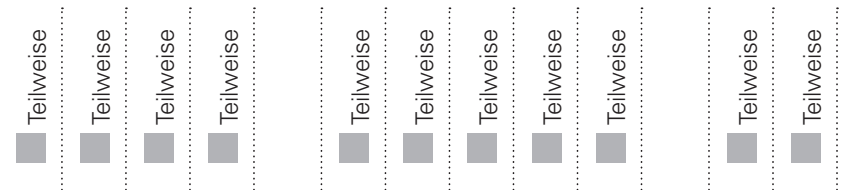

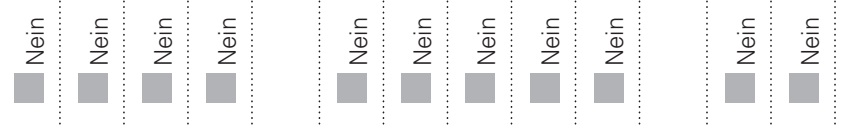

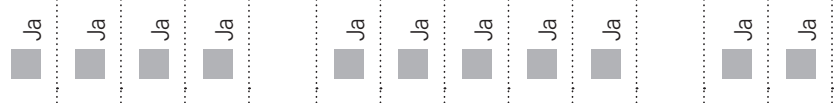

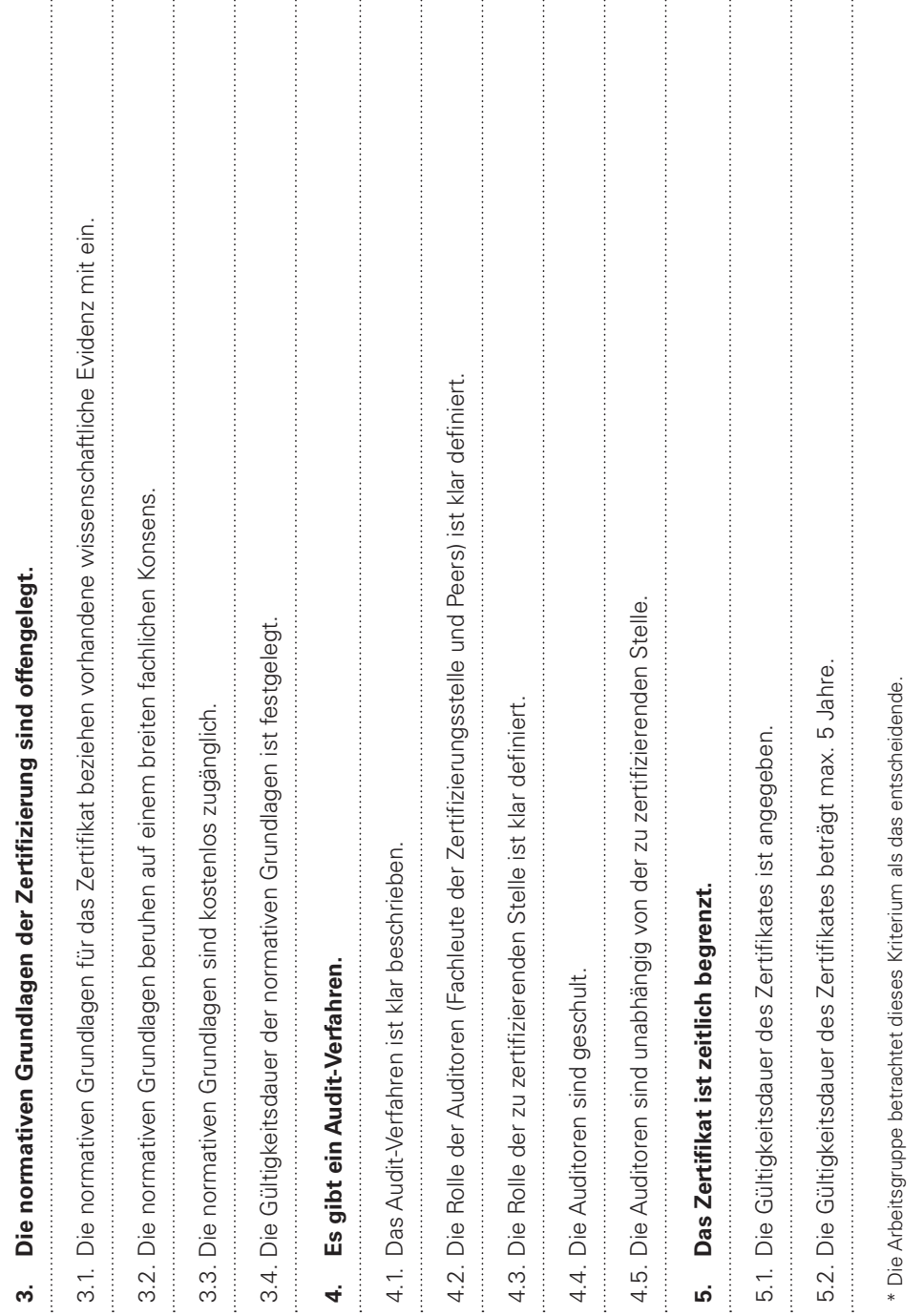


Anhang B: Gesetzliche Grundlagen

Folgende Artikel aus dem KVG und dem KVV befassen sich mit der Qualitätssicherung in der medizinischen Versorgung und stehen damit in einem Zusammenhang mit den vorliegenden Empfehlungen:

\section{KVG Art. 22a}

1 Die Leistungserbringer sind verpflichtet, den zuständigen Bundesbehörden die Daten bekannt zu geben, die benötigt werden, um die Anwendung der Bestimmungen dieses Gesetzes über die Wirtschaftlichkeit und Qualität der Leistungen zu überwachen. Namentlich sind folgende Angaben zu machen:

(...)

f. medizinische Qualitätsindikatoren.

\section{KVG Art. 58}

1 Der Bundesrat kann nach Anhören der interessierten Organisationen systematische wissenschaftliche Kontrollen zur Sicherung der Qualität oder des zweckmässigen Einsatzes der von der obligatorischen Krankenpflegeversicherung übernommenen Leistungen vorsehen.

2 Er kann die Durchführung der Kontrollen den Berufsverbänden oder anderen Einrichtungen übertragen.

3 Er regelt, mit welchen Massnahmen die Qualität oder der zweckmässige Einsatz der Leistungen zu sichern oder wiederherzustellen ist.

\section{KVV Art. 77}

1 Die Leistungserbringer oder deren Verbände erarbeiten Konzepte und Programme über die Anforderungen an die Qualität der Leistungen und die Förderung der Qualität. Die Modalitäten der Durchführung (Kontrolle der Erfüllung und Folgen der Nichterfüllung der Qualitätsanforderungen sowie Finanzierung) werden in den Tarifverträgen oder in besonderen Qualitätssicherungsverträgen mit den Versicherern oder deren Verbänden vereinbart. Die Bestimmungen haben den allgemein anerkannten Standards zu entsprechen, unter Berücksichtigung der Wirtschaftlichkeit der Leistungen. 
Akkreditierung formelle Anerkennung der Kompetenz einer Stelle (Organisation) für genau definierte Aufgaben.

Indikator Ein Indikator ist ein quantitatives Mass, das genutzt wird, um die Qualität der Führung, des Managements, der klinischen Unterstützungsfunktionen, die sich auf das Resultat der Leistungserbringung auswirken, zu monitorisieren und zu evaluieren.

Ein Indikator ist kein direktes Mass der Qualität. Er ist mehr ein Werkzeug, das zur Leistungsbewertung genutzt werden kann, das Aufmerksamkeit auf potenzielle Problembereiche lenken kann, die einer intensiven Überprüfung innerhalb der Organisation bedürfen könnten.

Dokument SAS 515 Leitfaden der SAS zur Beurteilung normativer Anforderungen an Managementsysteme als Grundlage für die Zertifizierung durch akkreditierte Zertifizierungsstellen. (www.seco.admin.ch/sas/00032/00077/index.html)

IS0/IEC 17021 Die Norm ISO/IEC 17021 wird in der Checkliste für die Akkreditierung von Zertifizierungsstellen von Managementsystemen definiert. (www.seco.admin.ch/sas/00032/00077/index.html)

Normative Dokumente Dokumente, die Regeln, Leitlinien oder Merkmale für Tätigkeiten oder deren Ergebnisse festlegen.

Normative Grundlage Gesamtheit aller Dokumente, welche die Anforderungen an ein Qualitätsmanagementsystem, ein Produkt oder eine Leistung beschreiben. Die Erfüllung dieser Anforderungen bildet die Grundlage für die Zertifizierung.

Medizinische Behandlungen Darunter werden alle Leistungen verstanden, die im Gesundheitswesen an Patientinnen und Patienten ambulant oder stationär erbracht werden. Die Leistungserbringung umfasst alle Prozesse am und mit dem Patienten, von der Abklärung zur Behandlung, Betreuung und Pflege durch interdisziplinäre und interprofessionelle Teams.

Wird die Leistung durch die koordinierte Zusammenarbeit mehrerer Leistungserbringer erbracht, sprechen wir von komplexen Behandlungssystemen oder Behandlungsketten.

Prüfprozess, Assessment, Audit Aussenstehende Dritte haben vor Ort zu prüfen, ob die normativen Anforderungen erfüllt werden. Wir sprechen von "Fremdbewertung", die Überprüfungsmethode ist das Audit. Diese Methode wird durch die Norm EN ISO 19011:2002 international geregelt.

Einzelne Zertifikate oder Labels werden ausschliesslich aufgrund einer "Selbstbewertung» (z. B. mittels Ausfüllens einer Checkliste oder eines Fragebogens) erteilt. Hier entfällt der Prüfprozess, wie inn die Arbeitsgruppe der SAMW postuliert.

Peer Ein Peer ist ein "Gleichrangiger» oder "Ebenbürtiger». Die Gleichrangigkeit des Peers bezieht sich sowohl auf die hierarchische Stellung in einer Einrichtung als auch auf die fachliche Kompetenz und den beruflichen Erfahrungshintergrund. Übernimmt der Peer die Funktion des Auditors, ist eher Gewähr für fachgerechte und inhaltlich vertiefte Prüfung, Empathie auf seiten des Auditteams sowie Akzeptanz der Bewertung auf seiten der Auditierten geboten.

Qualitätslabel Ein Qualitätslabel oder Qualitätsgütesiegel ist ein Konformitätszeichen, das bestätigt, dass ein Produkt oder eine Leistung bestimmte normative Anforderungen erfüllt. Ob ein Produkt oder eine Leistung die normativen Anforderungen erfüllt, wird durch ein Audit vor Ort überprüft. 
SAS Die Schweizerische Akkreditierungsstelle (SAS) ist im Staatssekretariat für Wirtschaft (seco) angesiedelt. Gemeinsam mit den potentiellen zu akkreditierenden Kunden, dem BSV, dem BFS, der GDK, der FMH und dem Organ für Akkreditierung und Qualitätssicherung $(\mathrm{OAQ})$ hat die SAS die Anforderungen an ein QM-System formuliert und 2002 in Kraft gesetzt.

Die SAS prüft kostenpflichtig in einer Vorprüfung die normative Grundlage der zu prüfenden Organisation. Wenn die Vorprüfung überstanden ist, erfolgt eine Akkreditierung nach international gültigen Regeln (z.B. des internationalen Akkreditierungsforum (IAF) oder ISO CASCO sowie der European Cooperation for Accreditation (EA)).

Standard Ein medizinischer Standard ist eine einheitliche oder vereinheitlichte, weithin anerkannte und meist auch angewandte (oder zumindest angestrebte) Art und Weise, etwas herzustellen oder durchzuführen. Er gibt den jeweils aktuellen medizinisch wissenschaftlichen Erkenntnisstand unter Berücksichtigung praktischer Erfahrung und professioneller Akzeptanz wieder. Er wird aus einzelnen Forschungsergebnissen, Lehrmeinungen und institutionalisierten Expertenkommissionen gewonnen und ist niedergelegt in Originalpublikationen, wissenschaftlichen Übersichtsarbeiten und Lehrbüchern. Hieraus können sich verschiedene, aber auch gleichwertige Diagnose- und Behandlungswege ergeben. (Hessisches Ärzteblatt 12/2003; 640-642)

Validität Ein Indikator misst das, was er vorgibt zu messen.

Zertifikat Urkunde oder Bestätigung, die beschreibt, aufgrund welcher Prüfprozesse zu welchem Zeitpunkt und durch wen festgestellt worden ist, dass ein Qualitätsmanagementsystem, ein Produkt oder eine Leistung die definierten normativen Anforderungen zum Zeitpunkt des Audits erfüllte.

Zertifizierung Das Resultat einer Prüfung (Audit) durch einen unparteiischen Dritten, die die Konformität sowohl des Prozesses (Arbeitsabläufe) als auch der Resultate mit definierten Gütekriterien, mit anerkannten Standards und Normen für eine bestimmte Zeitperiode bestätigt.

Qualität Qualität ist ein Mass dafür, wie sehr Leistungen des Gesundheitswesens für Individuen oder ganze Bevölkerungsgruppen

- die Wahrscheinlichkeit erwünschter Gesundheitsergebnisse erhöhen und - dem aktuellen Fachwissen entsprechen.

Qualitätsdimensionen Die Leistungserbringung soll wirksam, sicher, rechtzeitig (hierin kommt die Zugangsproblematik zu den Leistungen des Gesundheitssystems zum Ausdruck), patientenzentriert, nützlich/effizient und chanchengleich für alle erfolgen.

QM-System Aufeinander abgestimmte Tätigkeiten zur Leitung und Lenkung einer Organisation bezüglich Qualität.

Qualitätspolitik Übergeordnete Absichten und Ausrichtung einer Organisation zur Qualität. 



\section{Impressum}

Gestaltung

Druck

1. Auflage

Bestelladresse vista point, Basel

Schwabe, Muttenz

1000 d, 600 f (Juni 2011)

SAMW

Petersplatz 13

$\mathrm{CH}-4051$ Basel

Tel.: +41612699030

Fax: +4161269 9039

E-mail: mail@samw.ch

Diese Empfehlungen sind auf der Website der SAMW (www.samw.ch) online abrufbar.

Die SAMW ist Mitglied der

Akademien der Wissenschaften Schweiz 

